\title{
EL RIEGO DE PRECISIÓN EN EL CULTIVO DE FRESA EN LA PROVINCIA DE HUELVA
}

\author{
Martín Arroyo, M. ${ }^{1}$, Fernández García, I. ${ }^{1}$, Gonzalez Perea, . $^{1}$, García Morillo, J. ${ }^{1}$, \\ Rodríguez Díaz, J.A. ${ }^{1}$, Camacho, E. ${ }^{1}$ y Montesinos, $P{ }^{1}$
}

1 Departamento de Agronomía, Área de Ingeniería Hidráulica. Universidad de Córdoba. Campus de Rabanales, Edificio Leonardo Da Vinci, 14071, Córdoba. E-mail: o02maarm@uco.es, g52fegai@uco.es, g72goper@uco.es, g62gamoj@uco.es, jarodriguez@uco.es, ecamacho@uco.es,pmontesinos@uco.es

\section{Resumen}

El cultivo de fresa es el cultivo con mayor repercusión económica y social de la provincia de Huelva, ya que España es el principal productor de fresa fresca de Europa y el segundo del mundo, situándose el $95 \%$ de esa producción en la provincia de Huelva. Sin embargo, la zona donde se desarrolla el cultivo, el entorno del Parque Nacional de Doñana, es una de las zonas de mayor sensibilidad ambiental de Europa y cualquier actividad que se desarrolle debe evitar impactos negativos en el mismo. De lo anterior se deduce la necesidad de buscar un equilibrio para que agricultura y medio ambiente puedan convivir en el entorno de Doñana. Este trabajo se centra precisamente en eso, en optimizar el uso del agua en la zona mediante la implantación del riego de precisión en el cultivo de fresa, que consiste en dar a la planta el agua que necesita en el momento adecuado. La implantación de un sistema de riego de precisión se ha llevado a cabo en la campaña 2013/2014 en una finca comercial de la zona Almonte-El Rocío, provincia de Huelva. Para ello se ha implantado un ensayo en 5 sectores distintos, cada uno con un tipo de emisor diferente, en el que se realizó una programación óptima del riego basada en el cálculo de las necesidades teóricas del cultivo, el análisis hidráulico del sector y el movimiento del agua en el suelo, y cuyo comportamiento se comparó con un sector testigo manejado por el agricultor. Estas técnicas permitieron ahorrar un $43 \%$ de agua, en las condiciones particulares de suelo, variedad y configuración de la red riego de la finca de ensayo, obteniéndose un ligero aumento de producción respecto al manejo tradicional del agricultor, lo que se tradujo en un incremento de la rentabilidad del cultivo y de una mejora de la sostenibilidad en la producción intensiva de fresas.

\section{1- Introducción, Objetivos}

España es el principal productor de fresa (Fragaria $x$ ananassa) fresca de Europa y el segundo del mundo, detrás de Estados Unidos, con una producción cercana a las 300000 t/año. De esta producción, el $95 \%$ se sitúa en la provincia de Huelva, que se ha convertido en la mayor concentración productora de fresas del mundo.

Por una parte, el cultivo de fresa es el cultivo con mayor repercusión económica y social en la zona, y por otra parte, la zona donde se desarrolla el cultivo, el entorno del Parque Nacional de Doñana, es una de las zonas de mayor sensibilidad ambiental de Europa, siendo el agua el elemento que vincula los ecosistemas (marismas, lagunas) y la economía (fresas, arrozales). La relación del agua con el territorio genera importantes tensiones y problemas ambientales, especialmente en épocas de escasez del recurso, y cualquier actividad que se desarrolle en este entorno debe ser respetuosa con el mismo, ser viable económicamente y tener un impacto social positivo. 
Las dotaciones previstas por las administraciones públicas del agua en dicha zona preocupan al sector fresero, por considerarlas insuficientes, y están generando importantes tensiones entre la Administración, los agricultores y el sector medioambiental. De ahí que deba lograrse una dotación consensuada que satisfaga los intereses de las tres partes.

Por otra parte, el diseño de muchas de las instalaciones de riego en fincas comerciales no se ha realizado siguiendo criterios técnicos. Así mismo, los emisores utilizados, cintas de riego, son emisores turbulentos donde el caudal varía con la presión y tienden a obstruirse. Las circunstancias anteriores junto con un manejo del riego por parte del agricultor basado en su experiencia, hace difícil el uso sostenible del agua de riego en el cultivo de fresa en una zona tan sensible ambientalmente como es el entorno de Doñana.

Como alternativa sostenible aparece el riego de precisión, que consiste en dar a la planta el agua que necesita en el momento adecuado. Para ello es necesario conocer tanto los requerimientos de agua del cultivo como el movimiento del agua a través del suelo, muy condicionado por el alto contenido de arena, normalmente superior al $90 \%$ en la zona.

El objetivo del presente trabajo es la implantación de un riego de precisión que aplique el agua de forma uniforme en toda la parcela, mediante eventos de riego de duración variable a lo largo de la campaña, de modo que el agua se mantenga en los primeros $40 \mathrm{~cm}$ del suelo, evitando la percolación. El tiempo de riego se determinada a partir de variables medibles, reduciéndose así la incertidumbre sobre la cantidad de agua aplicada.

\section{2- Materiales y métodos}

\subsection{Ensayo de riego de precisión}

Una vez establecida la importancia de disponer de un sistema de riego de precisión en el cultivo de la fresa, la metodología propuesta se ha aplicado en un sector de riego de una finca comercial.

La implantación del sistema de riego de precisión se ha llevado a cabo en la campaña 2013/2014 en una finca comercial de la zona Almonte-El Rocío, provincia de Huelva. La variedad cultivada donde se ha llevado a cabo el ensayo es Splendor, con una densidad de plantación de 65000 plantas/ha, y la textura del suelo es $92 \%$ arena, $4 \%$ arcilla y $4 \%$ limo. La fecha de plantación tuvo lugar el 01/10/2013, la cubrición con plástico se realizó el 03/11/2013, retirándose ese plástico el 16/05/2014 y el final de campaña fue el 27/05/2014.

Se ensayaron cinco emisores de riego diferentes, seleccionados y evaluados previamente en el laboratorio (Martín Arroyo, M., 2013), cada uno instalado en un macrotúnel independiente, cuyo comportamiento se comparó con el del agricultor. Cada macrotúnel se manejó como un sector de riego independiente de $255 \mathrm{~m}^{2}$, controlado por una electroválvula en cabecera donde se reciben las órdenes del programador de riego. Además cada sector dispone de un contador inteligente, que almacena en su memoria cuando y cuánta agua se consume en cada evento de riego. La dosis de agua aplicada se basa en una programación óptima de riego, basada en el cálculo de las necesidades teóricas del cultivo, el análisis hidráulico del sector mediante el uso del simulador hidráulico Epanet (Rossman, 2002) y el movimiento del agua en el suelo, simulado mediante el modelo Hidrus (García Morillo, J., 2014). Para el cálculo de las necesidades teóricas del cultivo se ha instalado una estación agroclimática en la parcela de ensayos, compuesta por dos piranómetros de silicio, uno dentro y otro fuera del macrotúnel, y un sensor de temperatura dentro del túnel; y para monitorizar el movimiento del agua en el suelo se han instalado 3 equipos de monitorización de humedad en el suelo, formado cada uno de ellos por 3 sondas de humedad capacitivas (FDR), instaladas a tres profundidades (12, 25 y $40 \mathrm{~cm}$ ), y conectadas a un datalogger que registra los datos cada 15 minutos en 3 macrotúneles (testigo, 2 y 4). 
Este ensayo se llevó a cabo durante la fase de desarrollo del cultivo, es decir, la fase de riego por goteo, siendo la aplicación de agua en las fases previas de alomado y plantación igual en todos los macrotúneles del ensayo, realizada por el agricultor según su criterio.

Respecto a la fertilización, se aplicó durante toda la campaña el programa de fertilización que seguía el agricultor, realizado un seguimiento de la fertilización aplicada, con el objetivo de calcular las unidades de fertilizante (UF) utilizadas y asegurar que no hay problemas por exceso o defecto de fertilizantes.

El esquema del ensayo de riego de precisión se puede ver en la Figura 1.

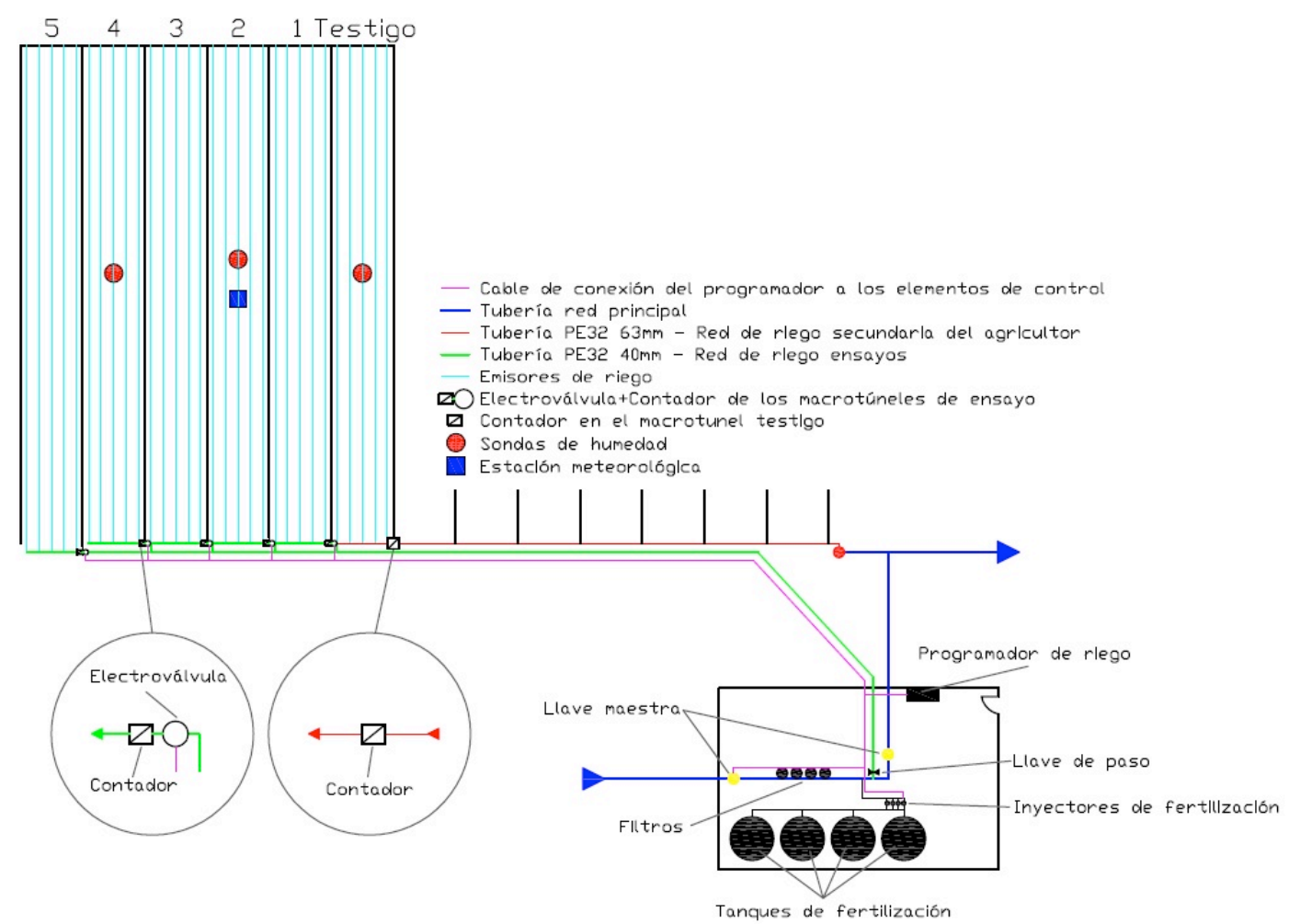

Figura 1. Esquema ensayo riego de precisión

Un aspecto fundamental al abordar el ensayo, es que el menor uso del agua de riego en ningún caso debe conllevar una pérdida de rentabilidad, por eso, además de medir cuando y cuánta agua se consume en cada uno de los 6 macrotúneles del ensayo, se registró la producción obtenida de primera y segunda categoría e industria, y se realizó un seguimiento de la calidad de la fruta obtenida, midiendo ${ }^{\circ} \mathrm{Brix}, \mathrm{pH}$, acidez, peso por unidad, forma, decoloración cuello, botrytis, sobremaduras, sabor y color visual, en cada uno de los macrotúneles del ensayo.

\subsection{Programación del riego}

Para realizar la programación del riego, en primer lugar se determinaron las necesidades de riego teóricas del cultivo, estimadas a partir de la serie histórica de datos climáticos (ETo) registrada en la estación agroclimática de la zona (Estación Meteorológica de Almonte, 2013) en las últimas 10 campañas (03/04 - 12/13), y de los coeficientes de cultivo (Kc) utilizados, que son los propuestos por FAO (Allen et Al., 2006) y posteriormente adaptados en el estudio de WWF para el cultivo de la fresa en Huelva (WWF, 2009) y en consonancia a los propuestos para la zona (Verdier, 1987). En segundo lugar, se determinó el tiempo de riego diario, a partir de las necesidades de riego teóricas del cultivo calculadas 
previamente, y del análisis hidráulico del sector realizado con el simulador Epanet (Tabla 1). El tiempo de riego varía en cada sector o macrotúnel, en función del caudal del emisor y de su rendimiento de aplicación $(\mathrm{Ra})$, el cual es función de la configuración hidráulica del sistema de riego. De esta forma se obtuvieron los tiempos de riego en minutos/día, repartidos en uno o varios pulsos de riego para minimizar la percolación. El número de pulsos se determinó mediante el modelo Hidrus. Estos tiempos de riego se corregían semanalmente con los datos registrados por una parte, en la estación agroclimática instalada en la parcela de ensayo, y por otra con los datos de sondas de humedad capacitivas (FDR). Por último se estableció una presión en cabeza del sector, resultante del análisis hidráulico (Tabla 1), que permitía trabajar bajo condiciones ideales de funcionamiento.

Tabla 1. Parámetros del comportamiento del riego

\begin{tabular}{|c|c|c|c|c|c|c|c|c|}
\hline Túnel & Tipo de emisor & $\begin{array}{c}\text { Dato } \\
\text { técnico }\end{array}$ & $\begin{array}{c}\text { Caudal } \\
\text { unitari } \\
\left(I \cdot \mathrm{h}^{-1} \cdot \mathrm{m}^{-1}\right)\end{array}$ & $\begin{array}{c}\text { Presión en } \\
\text { cabeza } \\
(\text { bar })\end{array}$ & $\begin{array}{c}\text { Caudal } \\
\text { del sector } \\
(\mathrm{I} / \mathrm{h})\end{array}$ & $\mathrm{CVq}$ & $\begin{array}{c}\text { Us } \\
(\%)\end{array}$ & $\begin{array}{c}R a \\
(\%)\end{array}$ \\
\hline 1 & Streamline 16080fl 3.6 & $\mathrm{C}$ & 3,6 & 0,85 & 758,96 & 0,032 & 96,8 & 94,3 \\
\hline 2 & Streamline 16080fl 5.25 & C & 5,25 & 0,85 & 1121,7 & 0,032 & 96,8 & 94,3 \\
\hline 3 & Dripnet PC 16200 & PC & 3 & 0,42 & 677,91 & 0,022 & 97,8 & 96,2 \\
\hline 4 & Amnon PC CNL & PC CNL & 3,67 & 1,1 & 825,62 & 0,042 & 95,8 & 92,7 \\
\hline 5 & Uniram As 17012 & PC AS & 5,33 & 0,65 & 1205,41 & 0,022 & 97,8 & 96,2 \\
\hline
\end{tabular}

$\mathrm{C}=$ Cinta de riego; $\mathrm{PC}=$ Autocompensante $; \mathrm{PC} \mathrm{CNL}=$ Autocompensante $\mathrm{y}$ antidrenante; $\mathrm{PC} \mathrm{AS}=$ Autocompensante y antisucción

El esquema integral de programación del riego se muestra en la Figura 2.

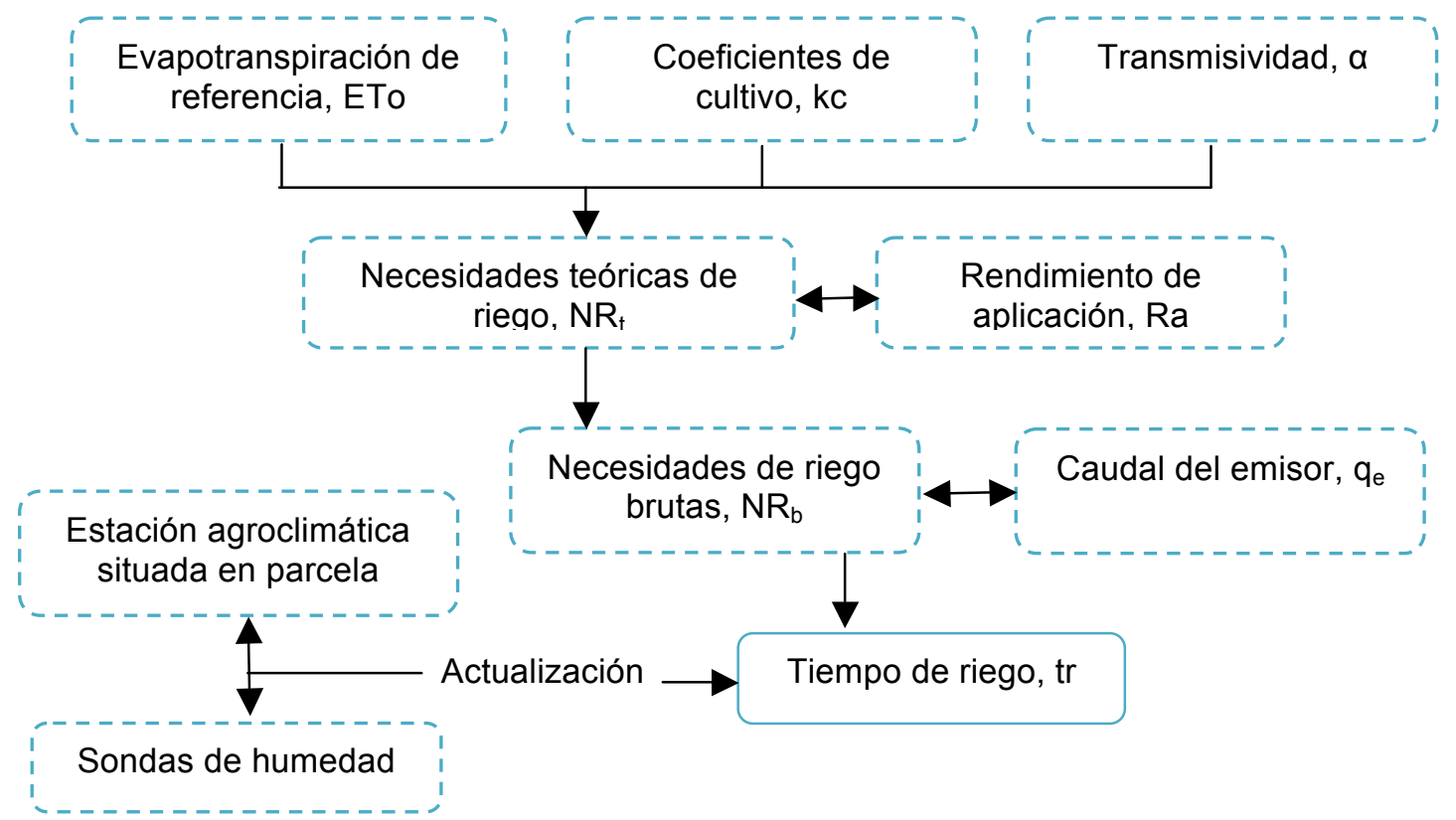

Figura 2. Esquema integral de programación del riego

\subsection{Análisis coste-beneficio}

La monitorización tanto del consumo de agua como de la producción permitió realizar un análisis coste-beneficio, en el que se calculó el aumento de rentabilidad en los macrotúneles con riego de precisión respecto al testigo. Para ello se tuvieron en cuenta, por una parte, los costes de producción tanto en el macrotúnel testigo como en los regados 
mediante el sistema de precisión, y por otra parte, el aumento de producción asociado a la producción que se obtuvo en los macrotúneles experimentales respecto al testigo. Los costes productivos considerados eran los asociados al sistema de riego, agua, fertilizante, energía en el cabezal de riego y mano de obra requerida para el riego y la fertilización. La estimación del aumento de beneficio logrado en los 5 macrotúneles donde se llevó a cabo el riego de precisión se calculó considerando los incrementos/decrementos mensuales de la producción en relación al testigo. Estos valores multiplicados por los precios medios mensuales menos el coste de recolección $(0,4 € / \mathrm{kg})$, que era el coste que cambiaba entre túneles, proporcionó el aumento de beneficio. Los precios medios mensuales de la campaña fueron: diciembre y enero, $1,93 € / \mathrm{kg}$; febrero, $1,13 € / \mathrm{kg}$; marzo, $0,8 € / \mathrm{kg}$; abril, $0,47 € / \mathrm{kg}$ y mayo, $0,36 € / \mathrm{kg}$.

\section{3- Resultados y discusión}

\subsection{Necesidades teóricas y brutas de riego}

Las necesidades teóricas del cultivo, coinciden con la ETc $\left(\mathrm{m}^{3} / \mathrm{ha}\right)$ al no intervenir la lluvia en el balance hídrico, y el consumo de agua en la fase de riego por goteo $\left(\mathrm{m}^{3} / \mathrm{ha}\right)$ para los diferentes túneles del ensayo y para los diferentes meses de la campaña aparecen en la Tabla 2.

Tabla 2. ETc y Consumo de agua en goteo

\begin{tabular}{|c|c|c|c|c|c|c|c|}
\hline \multirow{2}{*}{ Mes } & ETc & \multicolumn{5}{|c|}{ Consumo de agua en riego por goteo $\left(\mathrm{m}^{3} / \mathrm{ha}\right)$} \\
\cline { 3 - 8 } & $\left(\mathrm{m}^{3} / \mathrm{ha}\right)$ & Túnel 1 & Túnel 2 & Túnel 3 & Túnel 4 & Túnel 5 & Testigo \\
\hline Octubre & 163,4 & 173,2 & 173,2 & 169,8 & 176,2 & 169,8 & 197,9 \\
Noviembre & 247,6 & 308,0 & 296,2 & 274,2 & 263,0 & 270,3 & 565,9 \\
Diciembre & 186,7 & 250,6 & 234,9 & 223,2 & 203,6 & 211,4 & 477,7 \\
Enero & 156,1 & 195,8 & 191,9 & 188,0 & 168,4 & 176,2 & 379,8 \\
Febrero & 279,8 & 301,5 & 325,0 & 325,0 & 309,3 & 305,4 & 536,5 \\
Marzo & 450,5 & 477,7 & 481,6 & 540,4 & 524,7 & 591,3 & 873,2 \\
Abril & 664,3 & 787,1 & 822,3 & 865,4 & 716,6 & 724,4 & 1210,0 \\
Mayo & 911,0 & 998,5 & 967,2 & 1135,6 & 982,8 & 1002,4 & 1879,6 \\
\hline TOTAL & 3059,4 & 3492,4 & 3492,4 & 3721,5 & 3344,6 & 3451,3 & 6120,5 \\
\hline
\end{tabular}

El consumo de agua en la fase de riego por goteo en los 5 macrotúneles donde se realizó un riego de precisión varió entre los $3344,6 \mathrm{~m}^{3} /$ ha del macrotúnel 2 y los 3721,5 $\mathrm{m}^{3} /$ ha del macrotúnel 3 , mientras que el consumo del testigo en el mismo periodo fue de $6120,5 \mathrm{~m}^{3} / \mathrm{ha}$. Es importante destacar que las necesidades brutas de riego aplicadas en los 5 túneles de ensayo, tan próximas a las necesidades de riego teóricas, se debieron, principalmente al tipo de suelo (>90\% de arena y presencia de una capa de arcilla a $50 \mathrm{~cm}$ de la superficie). En otros suelos, con mayores profundidades de la capa de arena, las necesidades brutas de agua aumentan. Así mismo, longitudes de lomo inferiores a $45 \mathrm{~m}$, con pequeñas pérdidas de carga, permiten un buen comportamiento de las cintas de riego (túneles 1 y 2), a pesar de ser emisores no compensantes. El riego de precisión consiguió un ahorro medio de agua del $43 \%$ respecto al testigo en los 5 macrotúneles monitorizados. Si se tiene en cuenta el consumo de $657 \mathrm{~m}^{3} /$ ha registrado en la fase de alomado, y los 603 $\mathrm{m}^{3} /$ ha en plantación, el consumo total de los 5 túneles de ensayo varió entre $4604,6 \mathrm{~m}^{3} / \mathrm{ha}$ en el macrotúnel 4 y $4981,5 \mathrm{~m}^{3} /$ ha en el macrotúnel 3 , mientras que el consumo total del testigo fue de $7380,5 \mathrm{~m}^{3} / \mathrm{ha}$.

Para la fertilización de los macrotúneles experimentales se siguió el mismo programa de fertilización del agricultor, aplicando la misma solución fertilizante en todos los macrotúneles, para analizar así la influencia del riego de precisión en el proceso de producción de fresa. 
Durante toda la campaña se comprobó semanalmente que el programa de fertilización del agricultor no generaba problemas nutricionales en las plantas de los túneles de ensayo.

Las Figuras 3, 4 y 5 muestran los resultados obtenidos en la monitorización del riego en los 3 macrotúneles donde estaban instaladas las sondas de humedad, desde el $12 / 12 / 2013$ (día en que se instalaron las sondas) hasta el 27/05/2014 (final de campaña). Los sensores de humedad están a la profundidad del sistema radicular de la fresa en el periodo inicial $(12 \mathrm{~cm})$ y cuando alcanza su máximo desarrollo supera los $25 \mathrm{~cm}$. El tercer sensor se instaló a $40 \mathrm{~cm}$ ya que la raíz de este cultivo no suele alcanzar esa profundidad y, por tanto, un contenido de humedad elevado en este nivel indicaría pérdidas por percolación.

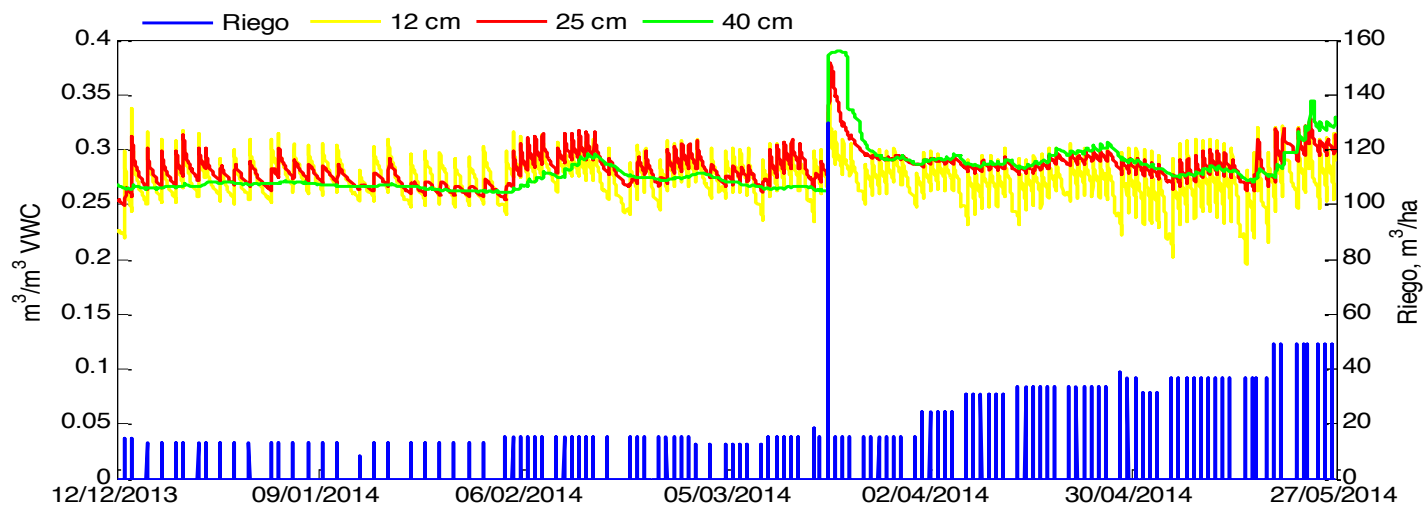

Figura 3. Monitorización del riego Túnel 2

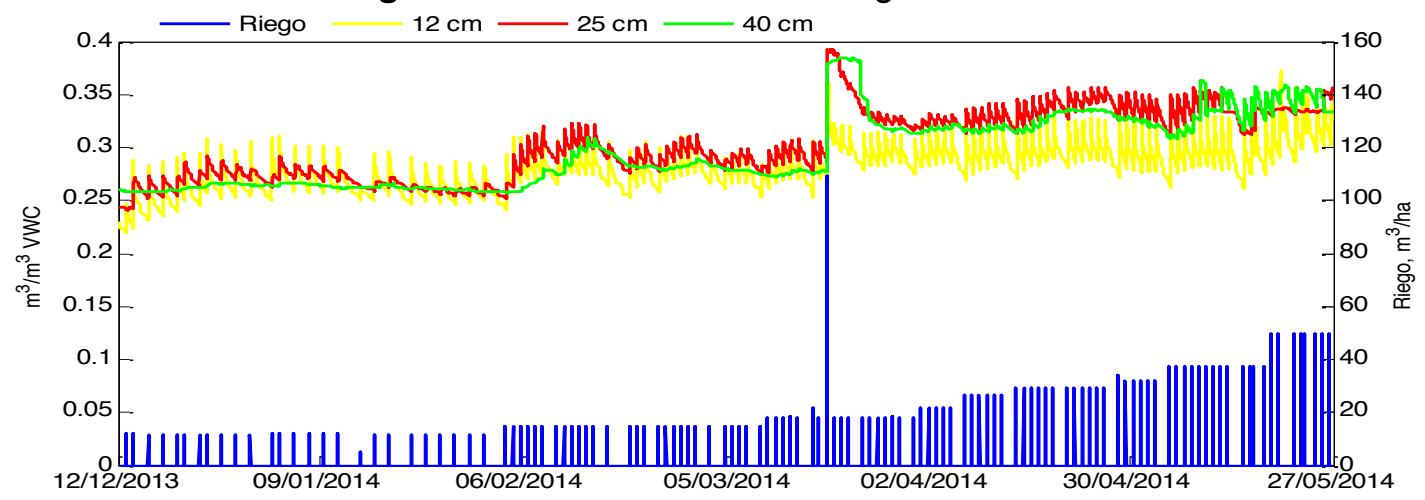

Figura 4. Monitorización del riego Túnel 4

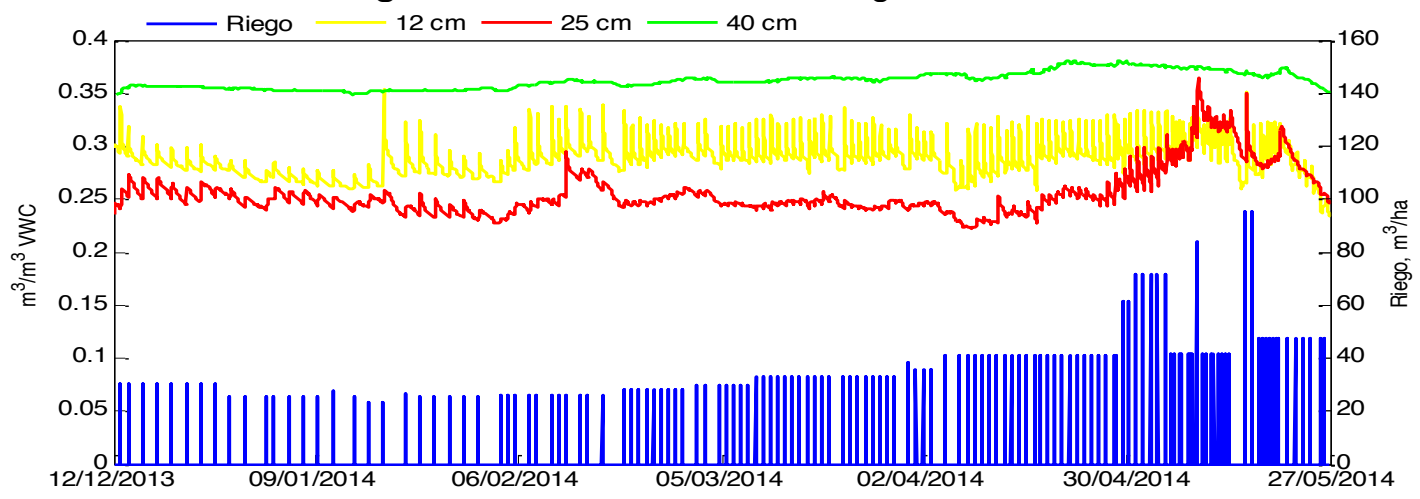

Figura 5. Monitorización del riego Túnel testigo

En el macrotúnel 2 y 4 (Figuras 3 y 4), los valores registrados por los sensores de humedad demuestran que la programación del riego ha sido adecuada. El contenido de humedad a 12 y $25 \mathrm{~cm}$ se mantiene siempre entre 0,25 y $0,3 \mathrm{~m}^{3} / \mathrm{m}^{3}$ VWC (volumen water 
capacity), excepto durante los días posteriores al fallo que se produjo en el programador del riego el 19/03/2014. El contenido de agua a $40 \mathrm{~cm}$ se situó en torno a $0,27 \mathrm{~m}^{3} / \mathrm{m}^{3}$ VWC durante toda la campaña hasta el día en el que tuvo lugar el fallo en el programador de riego. A partir de ese día, el contenido de agua alcanzó un valor de $0,29 \mathrm{~m}^{3} / \mathrm{m}^{3}$, ya que la presencia de una capa de arcilla próxima a la superficie dificultaba el drenaje del terreno. En las últimas fases del cultivo, para evitar percolaciones era recomendable aplicar varios pulsos de riego al día de menos de 30 minutos, lo cual no pudo realizarse por limitaciones técnicas en la finca de ensayos.

Con respecto al macrotúnel testigo (Figura 5), el contenido de agua a $12 \mathrm{~cm}$ se mantuvo en torno a $0,3 \mathrm{~m}^{3} / \mathrm{m}^{3}$ durante toda la campaña, excepto en la última semana, donde la retirada del plástico y el aumento de la temperatura dieron lugar a una reducción en el contenido de humedad en el suelo $\left(0,25 \mathrm{~m}^{3} / \mathrm{m}^{3}\right)$. Por tanto, el comportamiento fue similar al de los túneles 2 y 4 . Sin embargo, el contenido de humedad registrado a $25 \mathrm{~cm}, 0,25 \mathrm{~m}^{3} / \mathrm{m}^{3}$, fue inferior al obtenido en los túneles experimentales debido al tipo de emisor empleado en esta finca (cinta exudante). A $40 \mathrm{~cm}$, el contenido de humedad se mantuvo siempre por encima de $0,35 \mathrm{~m}^{3} / \mathrm{m}^{3}$, lo que indica que el suelo se encontraba en condiciones de saturación.

\subsection{Producción y calidad}

Se realizó un seguimiento de la producción obtenida de primera y segunda categoría e industria en cada uno de los macrotúneles del ensayo (Tabla 3).

Tabla 3. Producción

\begin{tabular}{|c|l|c|c|c|c|c|c|}
\hline \multicolumn{2}{|c|}{ Categoría } & \multicolumn{7}{|c|}{ Producción } \\
\cline { 2 - 8 } & Túnel 1 & Túnel 2 & Túnel 3 & Túnel 4 & Túnel 5 & Testigo \\
\hline \multirow{2}{*}{ Primera } & $\mathrm{kg} / \mathrm{ha}$ & 38027,2 & 37011,2 & 38683,0 & 36552,0 & 35659,8 & 36310,2 \\
& $\mathrm{~g} /$ planta & 585,0 & 569,4 & 595,1 & 562,3 & 548,6 & 558,6 \\
\hline \multirow{2}{*}{ Segunda } & $\mathrm{kg} /$ ha & 2701,9 & 3034,7 & 2897,6 & 2838,9 & 3093,4 & 2662,7 \\
& $\mathrm{~g} /$ planta & 41,6 & 46,7 & 44,6 & 43,7 & 47,6 & 41,0 \\
\hline \multirow{2}{*}{ Industria } & $\mathrm{kg} /$ ha & 11708,0 & 11629,7 & 11747,2 & 11747,2 & 11668,9 & 11747,2 \\
& $\mathrm{~g} /$ planta & 180,1 & 178,9 & 180,7 & 180,7 & 179,5 & 180,7 \\
\hline \multirow{2}{*}{ TOTAL } & $\mathrm{kg} /$ ha & 52437,1 & 51675,6 & 53327,8 & 51138,1 & 50422,1 & 50720,1 \\
& $\mathrm{~g} /$ planta & 806,7 & 795,0 & 820,4 & 786,7 & 775,7 & 780,3 \\
\hline
\end{tabular}

La producción total registrada en los 6 macrotúneles, aunque sin diferencias significativas, fue ligeramente superior en los macrotúneles 1, 2, 3 y 4 que la del testigo, mientras que la producción del macrotúnel 5 estuvo ligeramente por debajo. Si esta producción total se estudia por meses, la producción registrada en enero y febrero en los macrotúneles de ensayo fue superior a la registrada en el testigo, es decir, este aumento de producción se produjo en los meses en los que la fresa tiene los precios más elevados. Para el resto de meses (marzo, abril y mayo) la producción en los 6 macrotúneles fue idéntica. Esto se debe, entre otras razones, a que al seguir el mismo programa de fertilización del agricultor, y aplicar las mismas unidades de fertilizante, la planta, en los primeros meses de recolección tenía mayor disponibilidad de nutrientes al no producirse lixiviado de ellos, de ahí que la producción en estos meses fuera mayor que la registrada en el testigo. Respecto a la producción por categoría no hubo ninguna diferencia, siendo el porcentaje de fruta destinado a primera categoría, segunda categoría e industria igual en todos los macrotúneles.

Aparte del registro de la producción, también se llevó un seguimiento de la calidad de la fruta obtenida en los macrotúneles del ensayo, donde se medía ${ }^{\circ} \mathrm{Brix}, \mathrm{pH}$, acidez, peso por unidad, forma, decoloración cuello, botrytis, sobremaduras, sabor y color visual. El 
análisis de estos parámetros a largo de la campaña.no mostró diferencias significativas en la calidad de la fruta de los 5 macrotúneles con riego de precisión respecto a la del túnel testigo.

\subsection{Análisis de coste-beneficio}

El análisis de coste-beneficio realizado (Tabla 4) muestra los costes de producción anuales (fijos y variables) en todos los macrotúneles del ensayo, el aumento de beneficio y el aumento de rentabilidad obtenido en los macrotúneles donde se realizó el riego de precisión respecto al macrotúnel testigo.

Tabla 4. Análisis costes-beneficio

\begin{tabular}{|l|c|c|c|c|c|c|}
\hline \multicolumn{7}{|c|}{ Costes $\left(€ \cdot\right.$ ha $^{-1} \cdot$ año $\left.^{-1}\right)$} \\
\hline Tipo de coste & Túnel 1 & Túnel 2 $^{-1}$ & Túnel 3 & Túnel 4 & Túnel 5 & Testigo \\
\hline Sistema de riego & 399 & 399 & 357 & 453 & 325 & 399 \\
Agua & 870 & 870 & 898 & 853 & 865 & 1186 \\
Fertilizante & 1220 & 1220 & 1220 & 1220 & 1220 & 1220 \\
Energía cabezal de riego & 238 & 179 & 258 & 212 & 168 & 203 \\
Mano de obra riego y fertilización & 1107 & 1107 & 1205 & 1205 & 1205 & 1107 \\
\hline TOTAL COSTES & 3835 & 3776 & 3937 & 3942 & 3783 & 4115 \\
\hline \multicolumn{7}{|c|}{ Aumento de beneficio $\left(€ \cdot\right.$ ha $^{-1} \cdot$ año $\left.^{-1}\right)$} \\
\hline Producción & 1136 & 780 & 545 & 863 & 996 & 0 \\
\hline TOTAL AUMENTO DE INGRESOS & 1136 & 780 & 545 & 863 & 996 & 0 \\
\hline \multicolumn{7}{|c|}{ Aumento de rentabilidad $\left(€ \cdot\right.$ ha $^{-1} \cdot$ año $\left.^{-1}\right)$} \\
\hline AUMENTO DE RENTABILIDAD & 1416 & 1119 & 723 & 1036 & 1328 & 0 \\
\hline
\end{tabular}

Respecto a los costes, tampoco existen diferencias significativas entre los macrotúneles experimentales y el testigo. El coste medio anual asociado a los cinco macrotúneles experimentales fue de $3855 € \cdot \mathrm{ha}^{-1} \cdot$ año $^{-1}$ y el del testigo $4115 € \cdot \mathrm{ha}^{-1} \cdot$ año $^{-1}$, es decir, hubo una reducción media de costes en los macrotúneles donde se aplicaó un riego de precisón de $260 € \cdot h a^{-1} \cdot a_{n} o^{-1}$ respecto al testigo. Esta reducción de costes se debió principalmente a la reducción en el consumo de agua. En este ensayo, al utilizar las mismas unidades de fertilizante el menor uso de agua no lleva asociado un menor uso del fertilizante y el coste asociado al fertilizante fue el mismo en todos los macrotúneles, sin embargo, en otras fincas con otras estrategias de fertilización, el menor uso del agua sí lleva asociado la reducción del fertilizante, lo que conllevará un ahorro considerable.

Aunque la producción fue similar en los 6 macrotúneles, los macrotúneles experimentales tuvieron mayor producción a principios de campaña, cuando los precios son generalmente más altos. El incremento medio de beneficio en los 5 macrotúneles experimentales respecto al testigo fue de $864 € \cdot \mathrm{ha}^{-1} \cdot \mathrm{año}^{-1}$.

Teniendo en cuenta la reducción de costes y el aumento de beneficio que se produjo en el cambio del manejo y/o sistema de riego respecto al del túnel testigo, se obtuvo un aumento de rentabilidad medio de $1124 € \cdot \mathrm{ha}^{-1} \cdot$ año $^{-1}$ en los 5 macrotúneles experimentales, que extrapolándolo a una finca de tamaño medio en la zona (15 ha) podría suponer un beneficio adicional de $16860 € \cdot a^{n} o^{-1}$. Estas estimaciones pueden variar de un año a otro (cambio de variedad, problemas de plagas y enfermedades, situación del mercado, etc...), pero son una referencia a tener en cuenta.

\section{4- Conclusiones y recomendaciones}

La disminución de la disponibilidad de agua y el impacto ambiental de la producción intensiva de fresas, ligados a la variabilidad del mercado están forzando al sector fresero a 
realizar un uso eficiente de los recursos. Su futuro va a depender, en gran medida, de la implantación de sistemas de riego de precisión en las fincas de cultivo, que permitan una utilización más eficiente de recursos como el agua, energía o fertilizante. No obstante, estos sistemas deben permitir mantener los niveles de producción utilizando menos recursos productivos, mejorando de esta forma la rentabilidad de las explotaciones y minimizando el impacto ambiental de esta actividad al disminuir la aportación de elementos contaminantes al acuífero subyacente. Un manejo preciso del riego determina el momento, la frecuencia y el tiempo de riego adecuados según las características del cultivo, red de riego, clima y suelo. La implantación de sistemas de riego de precisión es fundamental para garantizar un uso sostenible del agua.

Este trabajo muestra que gracias a la implantación de un riego de precisión se ha reducido el consumo de agua un $43 \%$ de agua, en una explotación comercial, dadas sus características específicas (variedad, suelo, topología de al red, etc..) aumentando la rentabilidad del cultivo y mejorando su sostenibilidad.

En la presente campaña 2014/2015, se continúa realizando ensayos de riego de precisión en fincas comerciales con diferentes características (suelo, variedades y configuraciones de la red de riego, entre otros aspectos) y se está asesorando en la gestión del riego, implementando este sistema de riego en cerca de 1500 ha de fresa $(23 \%$ de la superficie total de fresa), de forma particularizada para cada finca.

\section{5- Agradecimientos}

El ensayo del sistema de riego de precisión se ha desarrollado en el marco del proyecto "Reducing the hydrological impact of the imported strawberries - Huelva región of Spain", financiado por la Fundación Coca-Cola.

\section{6- Bibliografía}

Allen, R. G.; Pereira, L. S.; Raes D.; Smith, M. (2006). Guías para la determinación de los requerimientos de agua de los cultivos. Estudio FAO Riego y drenaje 56.

Bralts, V.F. and Kesner, C.D. (1983). Drip irrigation Field Uniformity Estimation. Transactions of the Amer. Soc. Ag. Eng. 26(5):1369-1374.

Fundación Doñana 21. (2006). Manual de Buenas Prácticas Agrarias Sostenibles.In: De la Agricultura al desarrollo Rural Sostenible, Available at http://www.donana.es/wp content/uploads/2013/01/Manual-Buenas-practicas-agrarias-sostenibles.pdf.

J. García, M. Martín, E. Camacho, J.A. y P. Montesinos. (2014). Hacia el riego de precisión en el cultivo de la fresa. I Symposium Nacional de Ingeniería Hortícola. La agromótica en la Horticultura. Orihuela (Alicante). SECH.

Junta de Andalucía. (2013). Estación Meteorológica de Almonte. http://www.juntadeandalucia.es/agriculturaypesca/ifapa/ria/servlet/FrontController?actio $\mathrm{n}=$ Static $\&$ url=coordenadas.jsp\&c_provincia $=21 \& \mathrm{c} \_$estacion $=10$

Martín Arroyo, M., Montesinos, P., García Morillo, J., Rodríguez Díaz, JA., Camacho, E. (2013). Influencia de los emisores de riego en la sostenibilidad del uso del agua en el entorno del Parque Natural de Doñana. Actas del XXI congreso nacional de riegos. Orihuela (Alicante). C-13.

Martín Arroyo, M., (2013). Influencia del Riego en la Sostenibilidad del Uso del Agua en el Cultivo de la Fresa en el Entorno del Parque Nacional de Doñana. (Influence of 
irrigation on water use sustainability in strawberry cultivation in the surroundings of Doñana National Park). Degree dissertation. UCO.

Martín Arroyo, M., Fernández García, I., González Perea, R., García Morillo, J., Rodríguez Díaz, J.A., Berbel, J., Camacho, E., Montesinos, P. (2014). Fundamentos del riego de la fresa en la provincia de Huelva. http://riegofresacocacola@blogspot.com.es

Rossman, L.A. (2002). "EPANET: User Manual. Risk Reduction Engineering Laboratory Office of Research and Development". United States Environmental Protection Agency. Cincinnati, $\mathrm{OH}$.

WWF. (2009). Manual de buenas prácticas de riego. Propuestas de WWF para un uso eficiente del agua en la agricultura. Viñedo, Olivar, Cítricos y Fresa. Madrid. Spain. 